\title{
Electromyography: A review of the current status of subvocal speech research
}

\author{
LINDA I. GARRITY \\ Mendota Mental Health Institute, Madison, Wisconsin 53704
}

\begin{abstract}
Electromyographic studies of subvocal speech are evaluated with two different aims: (1) a methodological review of controls for nonspeech artifact and of data analysis techniques, and (2) a review of substantive findings and suggestions for future research. A highly effective procedure, involving manipulation of phoneme content in certain trials, has been developed to control nonspeech artifact generated by the speech musculature. The commonly used data analysis technique of amplitude measurement of the single highest polygraph pen deflection is inadequate. Techniques which take frequency into account, such as analog computer integration of voltage values, are more sensitive. Even greater sensitivity is needed to isolate individual words so that rehearsal strategies can be investigated. It has been clearly established that preschool children as young as age 4 engage in spontaneous subvocalization that is related to recall in boys but not in girls.
\end{abstract}

The role of subvocal speech (also termed covert, silent, or inner speech) in memory and problem solving has been of interest for some time. Subvocal speech, a telegraphic or truncated version of social speech, is a form of peripheral speech-motor activity consisting of attenuated movements of the speech muscles. According to one notion, subvocal speech is regarded as the principal mechanism of thought (Vygotsky, 1962). According to another notion, subvocal speech is regarded at most as an index of, but not a causal factor in, thought (Flavell \& Hill, 1969). According to yet another viewpoint, subvocal speech is not at all related to thought, the latter occurring exclusively in the cortex. These various notions roughly correspond, respectively, to the peripheralist, moderately centralist, and centralist theories of thought, the pheripheralist theory placing the greatest importance on the external speech organs for their role in sending to the brain signals that play an important initiatory function in thought. (See McGuigan, 1970b, for a brief discussion.) The peripheralist and moderately centralist theories are of interest in this paper because they suggest that measurement of speech electromyography (EMG) will provide information about the thought process.

Measurement of speech EMG or subvocal speech is important because it provides a direct method of investigating the involvement of the peripheral speechmotor mechanism in establishing and maintaining speech code representations in short-term memory. It is commonly believed that information would most efficiently be transferred from short- to long-term

Sincere thanks go to John W. Giebink, Robert M. Leff, and Saul M Levin for the critical reading of this manuscript. Author's address: Mendota Mental Health Institute, 301 Troy Drive, Madison, Wisconsin 53704. memory by a linguistic process such as rehearsal (in addition to being indefinitely retained in short-term memory by rehearsal), since information in long-term memory is probably stored in a common linguistic format based upon the meaning of words or events. There is some debate as to whether information in short-term memory is stored primarily by acoustic (items that sound similar) or articulatory (items produced with a similar sequence of muscle patterns or kinesthetic cues) means. Unfortunately, it is difficult to distinguish between these two possibilities, since most words that sound alike tend to be produced in similar ways by the muscles; thus, the term "phonological" will be used to refer to these two linguistic means of encoding information.

Strong evidence for phonological information processing is provided by studies of errors in recall of visual as well as verbal items in that errors tend to be either acoustic or articulatory. If the peripheral speech-motor mechanism is primarily responsible for speech mediation, one would expect a decrease in the proportion of phonological errors when subvocalization is inhibited, because of interference with peripheral encoding. It should be noted, however, that use of a suppression technique to inhibit subvocalization may interfere with an as yet undefined central process whose effectiveness is reduced by the additional energy required to concentrate on reduction of subvocal activity.

Some speech EMG research exists on the role of articulation in producing phonological confusions in memory, but unfortunately, the results of these studies yield conflicting findings. Glassman (1972) presented data supporting a peripheral articulatory hypothesis, while the data of Cole and Young (1975) indicate that encoding of speech in short-term memory 
does not depend on subvocalization. In the Glassman (1972) study, experimental subjects were trained to inhibit their laryngeal speech EMG activity via auditory feedback, whereas control subjects' subvocal activity was simply "monitored." Half the subjects in each group received high- and half received low-acousticsimilarity three-word sets. The experimental group produced a smaller proportion of phonological errors than the control group. In the Cole and Young (1975) study, all subjects were trained to suppress laryngeal speech EMG activity, but only the experimental group was instructed to suppress subvocalization during the experiment. The task consisted of memorizing sequences of six consonant or vowel syllables. Analysis of the error patterns indicated a similar pattern of overall errors and intrusion errors both during high- and lowsubvocalization trials.

In addition to the suppression paradigm just discussed, the study of spontaneously occurring speech EMG can provide support for phonological involvement in memory insofar as a high correlation exists between occurrence of subvocal speech and recall. Several such studies will be described later.

In the present paper, the current status of EMG studies of subvocal speech is reviewed and suggestions are made for future directions. Only the more recent, better controlled EMG studies will be discussed. For other reviews of EMG studies of subvocal speech, see Edfeldt (1959), Locke (1970), McGuigan (1966, 1970b), and Sokolov (1969, 1972). Studies using adult subjects as well as relevant child studies will be reviewed.

EMG is a technique of recording electrical changes which accompany muscular activity through the use of bipolar electrodes. In a resting state, the inside of the muscle fiber has a negative and the outside a positive electrical charge. When a muscle's motor endplate receives impulses from the central nervous system via the motor nerves, these impulses, called action potentials, rapidly travel down the entire muscle membrane, causing a temporary reversal of the muscle's polarization. That is, the outside of the muscle fiber temporarily becomes negative and the inside positive, resulting in the muscle's contraction. In bipolar recording, the traveling negative charge reaches the nearer electrode first, causing the electrode to be negative with respect to the more distant electrode, and causing the polygraph pen to jump briefly above the resting baseline. Later, the traveling negative potential reaches the more distant electrode, causing it to be negative with respect to the first electrode, and causing the polygraph pen to briefly deflect below the resting baseline. (See Lippold, 1967, for more detail.)

Speech EMG has been recorded from a variety of muscles. In general, however, roughly similar results are obtained when subvocalization is measured from the chin, lip, tongue, or laryngeal speech musculature. Also, simultaneous recordings from different leads (i.e., chin and lip) have been found to be correlated (Garrity, 1975b). Faaborg-Andersen (1965) reviewed studies of the intrinsic and extrinsic laryngeal muscles and found that the muscle groups involved in normal speech and in inner speech were similar, although the amplitude of the latter was lower. The majority of studies have recorded from either the chin or lip muscles.

The use of adequate controls for nonspeech artifact is a central issue in EMG research as is the use of appropriately sensitive data analysis techniques. To be a useful source of data to study information processing, speech EMG measures must be relatively free of nonspeech artifact (or must adequately control for it) and must be sensitive enough to detect relatively small changes in activity level. This is particularly important when children are the subjects, since they tend to be more active, engage in more nonspeech artifact (e.g., lip licking and pursing, swallowing, and grimacing, in the case of lip recordings) and possibly have lower amplitude speech EMGs.

\section{CONTROLS FOR NONSPEECH ARTIFACT}

Three types of control procedures have been used to date: (1) Baseline EMG activity recorded while the subject is resting has been compared to EMG activity recorded during task performance. The major problem presented by the use of a resting baseline is that it does not control for the increased generalized tension levels subjects experience during task performance, which are associated with a variety of factors including increased difficulty. (2) Simultaneously recorded nonspeech muscle activity has been compared to speechmuscle activity. This procedure avoids the problem of experimental condition differences in generalized tension levels, but is not able satisfactorily to isolate nonspeech artifact from speech EMGs. (3) Trials or tasks containing words that involve little EMG activity when measured from a particular recording site (e.g., lips, tongue) have been compared to trials containing words requiring greater muscle activity when silently pronounced (e.g., nonlabial vs. labial or nonlingual vs. lingual words). This is the most satisfactory method used to date. It is a highly conservative control procedure in that "nonlabial" trials, for example, consist of only small speech gestures plus nonspeech artifact, while labial trials consist of the same artifact plus larger speech gestures. The assumption is made that nonspeech artifact is randomly, hence equally, distributed over labial and nonlabial trials. Studies involving each of the three control procedures will be reviewed and the factors associated with subvocal speech activity will be discussed.

\section{Resting Baseline}

Most studies have compared speech EMGs recorded during various task conditions to a resting baseline 
control condition. These studies may be grouped into those comparing: linguistic and nonlinguistic task performance, varying levels of difficulty on similar tasks, and subject populations on varying characteristics. In general, speech EMG activity has been found to vary in a predictable manner according to task and subject characteristics.

One approach has been to compare speech EMG activity during linguistic and nonlinguistic task performance. Speech EMGs have generally been greater during performance of linguistic (e.g., writing words) than of nonlinguistic (e.g., drawing ovals) tasks, even though covert speech could have occurred during performance of the nonlinguistic tasks (McGuigan, 1967b, 1970a). Furthermore, active visual linguistic tasks, such as reading silently or memorizing prose, tend to result in greater speech EMGs than do passive auditory linguistic tasks, such as listening to prose (McGuigan \& Bailey, 1969a). These findings, however, may be confounded, and actually attributable to the possible greater difficulty (leading to greater subvocalization) of the linguistic tasks, as borne out by Sokolov's (1969) research involving different difficulty levels of reading, arithmetic, simple counting, and visual tasks.

A related approach has been to compare speech EMG activity under varying levels of difficulty on similar tasks. In general, speech EMG activity has been found to increase with increasing task difficulty (Bassin \& Bein, 1961; Edfeldt, 1959; Faaborg-Andersen \& Edfeldt, 1958; Novikova, 1961; Sokolov, 1969), perhaps because more information is being processed (the easier tasks being more automatic). Also, tasks made more difficult by auditory background interference (i.e., presentation of prose) while subjects were reading led to greater speech EMG activity than when subjects read in silence (McGuigan \& Rodier, 1968).

Since amount of speech EMG can vary according to type of task, difficulty level, and so on, it is important to control for these potential confounds unless they are the variables under examination.

Another approach has been to compare speech EMG activity in differing speech populations. Slow readers (Edfeldt, 1959) and subjects with poor handwriting (McGuigan, 1967b, 1970a) engaged in more silent speech during silent reading or writing than did good readers and writers. The usual explanation of these findings has been that the task was more difficult for low-than for high-proficiency subjects. It is also plausible that silent speech may interfere with these activities.

In the only reported EMG longitudinal study, McGuigan, Keller, and Stanton (1964) found speech EMG amplitude decreased as children increased in age. In two experiments (McGuigan et al., 1964), either chin $(n=36)$ or lip $(n=60)$ EMGs of children aged 6 to 11 years were recorded while they read a series of passages of increasing difficulty. Amplitudes of the single highest polygraph pen deflections during reading, and during pre- and postreading resting baseline periods were measured for each passage. Speech EMGs in both studies were significantly higher during reading than during either pre- or postreading controls. There tended to be a positive relationship between frequency and amplitude of subvocalization. McGuigan and Bailey (1969b) retested 13 children after 2 years and the same 13 plus an additional 3 subjects after 3 years (at a mean age of 10 years). Although second- and thirdyear speech EMG amplitude was still higher during reading than during a resting baseline, it was significantly lower than during the original testing, and comparable to adult amplitudes.

In addition to the above studies with task and subject characteristics, a handful of studies have attempted experimentally to manipulate subvocal speech. Operant conditioning has been used in the attempt to decrease or inhibit subvocal speech through the use of noxious auditory feedback activated when speech EMGs reach a predetermined amplitude. Hardyck, Petrinovich, and Ellsworth (1966) were able to inhibit speech EMG activity, but found reading comprehension diminished as material increased in difficulty (Camacho, 1967; Hardyck \& Petrinovich, 1970; Hardyck, Petrinovich, \& Ellsworth, 1967). In subjects of below-average IQ, temporarily inhibited subvocal speech quickly returned, while in average or above-average IQ subjects, it remained at a decreased level up to 1 month later. Using an experimental and a control group, both initially trained to suppress subvocalization but only the experimental group required to suppress during the actual task, Cole and Young (1975) also found a recall decrement (in their experimental group). Conversely, Glassman (1972) found that the overall recall of a control group whose subvocal speech was not suppressed was similar to that of an experimental group trained to suppress subvocalization, although no statistical EMG data was presented to assure effectiveness of the suppression technique. The lack of a between-groups difference in overall recall may also be due to the possibility that the memory task was too easy.

In the studies discussed above, it should be noted that any comparison of a suppression with that of a nonsuppression group or condition is potentially confounded because (1) the task is more difficult for the suppression subjects in that they are required to concentrate not only on the memory task but also on their subvocalization process; (2) even if a significant decrease in subvocal activity is demonstrated, there is no guarantee that all relevant speech activity has been inhibited.

In a related approach, the task performance of subjects has been compared before and after they were trained or experimentally induced to subvocalize. Compared to a control group, McGuigan and Pinkney 
(1973) found increases in the reading rate (words per minute) of high school and college students and in the tongue EMG activity of college students after the two groups received speed-reading instruction. However, it appears that the increased EMG tongue activity of the college students in the speed-reading group may be related to the fact that they read larger quantities of material; the EMG activity was apparently indexed relative to fixed time intervals rather than to given amounts of reading material. Whether or not subvocal speech can be successfully induced or inhibited over time needs to be established; thus, the value of this line of investigation awaits more definitive research (McGuigan, 1967a).

Two crucial questions arise from the above findings: (1) Is the increase in speech-muscle activity over a resting baseline due to a generalized heightened activity level occurring during the more intense task-performance situation or is it specific to the speech musculature? (2) How much activity which is specific to the speech musculature is nonspeech related? This activity is often electromyographically indistinguishable from subvocal speech responses per se, and may increase along with them, for example, as a function of increasing task difficulty.

\section{Nonspeech Muscles}

One often used approach to the first question (i.e., controls for heightened generalized activity) has been the comparison of simultaneously recorded nonspeech activity (e.g., leg, nondominant arm, and finger EMGs) with subvocal speech activity both during task performance and resting baseline. In general, speech EMG has been found to occur independently of, and to increase more than, nonspeech EMG during task performance (Bassin \& Bein, 1961; McGuigan, 1967b, 1970a; McGuigan, Culver, \& Kendler, 1971; Sokolov, 1969). Other psychophysiological measures have also been compared to the speech EMG. Galvanic skin response (GSR) was found to occur independently of speech EMG (Sokolov, 1967), while respiration rate increased along with speech EMG during task performance (McGuigan, 1967b; McGuigan \& Bailey, 1969a; McGuigan et al., 1964; McGuigan \& Rodier, 1968).

Even more specifically, McGuigan and Winstead (1974) compared the tongue, lip, right- (preferred) arm, left-arm, and right-leg EMG activity of 12 adolescents during baseline, while reading sentences or while memorizing slides of words (either heavily bilabial or lingual-alveolar). Similar results were obtained for the reading and memorization tasks: Lip activity was greater on bilabial (involving both lips) and lower on lingual-alveolar (involving the tongue) tasks, indicating that the increase in speech-muscle activity over a resting baseline is not due to heightened generalized activity (as was preferred-arm EMG activity) but is quite specific to speech. Results for the left arm and right leg showed no clear pattern, whereas right-arm EMG increased above baseline on all tasks.

Using preschool children, Garrity (1975a) similarly found a strong relationship between EMG activity of two speech leads (chin and lip) and no relationship between this activity and that of a facial nonspeech control (temporalis) on either the labial or nonlabial trials of a short-term memory task. Along the same lines, deaf subjects with knowledge of both manual and oral speech have shown increases in both dominant hand and speech EMGs during problem-solving tasks, whereas only speech EMGs increased in normal-hearing subjects (McGuigan, 1971; Novikova, 1961).

Studies comparing speech-muscle activity with nonspeech physiological measurements have thus established that the activity recorded from the speech musculature is independent of heightened generalized activity level, with the possible exception of respiration rate.

\section{Words Varying in Speech EMG Activity}

The most effective method developed yet for discriminating speech from nonspeech artifact produced by the speech musculature was suggested by Locke and Fehr (1970a). Adult subjects' EMG activity while silently memorizing trials of labial words (e.g., words consisting of labial phonemes as $/ \mathrm{p} /, / \mathrm{b} /, / \mathrm{m} /, / \mathrm{f} /, / \mathrm{v} /)$ which involved a greater amount of lip movement was compared with low-labial phoneme trials (e.g., /c/, $/ \mathrm{g} /, / \mathrm{d} /, / \mathrm{t} /, / \mathrm{l} /)$ requiring minimal lip movement. The amplitude of the single highest pen deflection during each trial's stimulus presentation (visually presented words) and 10-sec prerecall delay periods was measured. During both periods, the amplitude during labial trials was found to be greater than during nonlabial trials.

Similar results using a similar procedure were found with 12 preschool children (Locke \& Fehr, 1970b). After brief pretraining, subjects were instructed to view silently three successively presented pictures until asked for recall. The amplitude of the single highest pen deflection during the five labial trials was higher than during the five nonlabial trials during the picture presentation, but not during the delay period. Recall data were not analyzed.

In a later study using a similar procedure, Locke (1971) compared EMG amplitudes of adults who read three passages of equal length containing $83 \%, 48 \%$, and $14 \%$ of labial words. Corresponding mean amplitudes were $36.2,21.2$, and 23.9 , with the highest labial passage having significantly greater amplitude than the two lower labial passages.

Similarly, Locke and Fehr (1971) found adult speech EMG amplitude to be greater for labial than for nonlabial lists of visually presented familiar words during recall, both when recall was written and when it was oral. In a study comparing two modes of stimulus 
presentation (visual and aural) and two recall methods (oral and written), Locke and Fehr (1972) found that adults emitted significant amounts of subvocal speech during the stimulus presentation only for the visual-stimulus/oral-recall and the aural-stimulus/writtenrecall conditions. During the prerecall delay period, significant amounts of subvocal speech were found for all four conditions. Subjects were informed of which stimulus and recall modes they would be using prior to each condition. These findings suggest that the occurrence of subvocal speech during the prerecall delay period is relatively invariant, in that it tends to be uninfluenced by the mode of stimulus presentation or recall.

To determine if amount of EMG activity or recall is differentially affected by pictures or visually presented words, Goldstein, Locke, and Fehr (1972) studied 26 school children in four conditions: labial pictures and words and nonlabial pictures and words. Amplitude was greater for labial than nonlabial trials, but no differences were found between pictures and words for all 26 children or for the 10 highest compared to the 10 lowest subvocalizers.

These studies isolate the relative contributions of nonspeech artifact from those of speech and provide a promising methodological approach, which can be used to answer more precise questions regarding the role of subvocal speech in information processing. Put differently, various of the above discussed techniques are important in that they provide checks on the validity of the obtained EMG data. Without the use of (1) nonlabial trials as a conservative baseline or (2) simultaneous recordings of nonspeech (preferably facial) muscles or other physiological functions, it would be difficult to determine if the speech EMGs are indeed attributable to speech.

\section{DATA ANALYSIS TECHNIQUES}

Two principal techniques have been used in studies of subvocal speech to date to analyze EMG data: measurement of the amplitude of the single highest (or several highest) polygraph pen deflection(s) per trial segment (e.g., stimulus presentation or delay periods), and analog computer routines for squaring and integrating voltage values over trial segments. The majority of subvocal speech studies reviewed in this paper have used the procedure of measuring the amplitude of the single highest pen deflection. This procedure, however, does not take into account the frequency of occurrence or density of high-amplitude signals and is therefore apt to rely more heavily upon the more infrequent but often very high-amplitude nonspeech artifact spikes generated by the speech musculature than measurement procedures which take frequency into account. Few subvocal speech studies to date have used the computer technique of squaring and integrating voltage values. This technique has been compared with the amplitude measurement procedure for the single highest pen deflection, as well as for the five highest pen deflections per trial segment. The results of these comparisons are discussed below.

Garrity (1975a) extended Locke and Fehr's (1970b) work using a different data analysis procedure and measuring recall. The lip-, chin-, and temporal-muscle EMG activity of 464 - and 5-year-olds was recorded while they silently viewed three successively presented pictures (10 trials) and waited $15 \mathrm{sec}$ to be asked for recall. In addition to the polygraph, EMG activity was recorded onto analog tape, the total voltage squared and integrated on an analog computer, and then converted to $T$ scores. $T$ scores for labial trials were higher than for nonlabial trials for the lip and chin but not the temporalis (facial) muscle, indicating artifact-free subvocal speech had occurred.

Recall also correlated with lip $(r=.46$ for picture presentation and $r=.50$ for the delay) and chin $(r=.44$ and .51$)$, but not temporalis activity $(r=-.09$ and .18$)$ for the 46 children. An unexpected significant correlation occurred between recall and subvocal speech for 15 boys $(r=.73$ for picture presentation and $r=.88$ for the delay period), but not for 15 girls similar in age, IQ, and recall $(\mathrm{r}=.32$, and .41$)$. Voltage values correlated higher with the five highest pen deflections (ranging from .40 to .85 for the trials) than with the single highest pen deflection (from .21 to .71) (Garrity, 1975b).

Similar results were found in a replication study (Garrity \& Donoghue, 1977), in which the lip EMG activity of 22 3-to 6-year-olds were recorded while they viewed four successively presented pictures on each of 12 trials. Labial trial EMG activity was greater than that on nonlabial trials. Significant correlations between recall and subvocal speech were found for boys $(r=.69$ for picture presentation and .72 for delay), but not for girls $(r=.55$ and -.10$)$. No differences in amount of subvocalization due to age or IQ were found.

\section{DISCUSSION}

In summary, within the last few years some very promising techniques have been developed for the control of nonspeech artifact. The earlier studies that relied solely upon activity recorded during a resting baseline period had no satisfactory means of estimating the degree to which their data were inflated by nonspeech artifact (such as grimacing, lip pursing, lip licking) emitted by the speech muscles during the more tense task-performance situation.

Earlier attempts to solve this problem centered around the comparison of speech EMGs with spontaneously recorded physiological measures such as nonspeech EMG, GSR, and so on. While this procedure addressed the problem of generalized heightened body 
activity related to the more tense task performance situation, it still could provide no information about the amount of nonspeech-related activity that was specific to the speech musculature or about the relation of speech EMG artifact to nonspeech physiological activity.

An answer to the above question has been provided by the more recent technique of using low EMG activity speech phonemes (nonlabial, nonlingual) during some trials as a control for speech phonemes that generate higher EMG activity when silently pronounced. With this conservative control procedure, the door has been opened to investigate a host of more intricate questions.

Similarly, in the area of data analysis techniques, suggestions have been made regarding more sensitive methods. A common procedure in EMG studies of subvocal speech has been measurement of the amplitude of the single highest polygraph pen deflection per task segment. However, this procedure does not take frequency into account and, therefore, probably incorporates relatively greater amounts of nonspeech artifact. Use of this relatively insensitive measurement procedure may be responsible, for example, for the lack of significant differences in recall between high and low subvocalizers in the Goldstein et al. (1972) study. Using the more sensitive procedure of squaring and integrating EMG voltages for the stimulus presentation and the delay periods of each trial, greater recall was found for higher than for lower subvocalizers (Garrity, 1975a). Noticeably higher correlations were obtained between integrated voltage values and the five highest pen deflections (correlations ranged from .40 to .87 ) than between integrated voltage values and the single highest pen deflections (correlations ranged from .21 to .72) (Garrity, 1975b). Thus, while integration of total EMG power is probably the most reliable method, if the necessary equipment is not available, amplitude measurement of the five (or several) highest pen deflections is more accurate than the single highest because frequency is to some degree taken into account.

The possibility of an even more inexpensive method, the use of observers trained in lip reading, has been suggested by Locke and Ginsberg (1975). These authors had two trained observers judge whether subjects were subvocalizing either high-labial or low-labial phonemes on a given trial. On all trials, the phoneme set consisted of the same four contiguously presented high-labial or the same four contiguous low-labial phonemes (a total of eight different phonemes was used). An auditory stimulus alerted the observers to the beginning of each four-phoneme set. The observers were able to correctly determine the labiality of each trial $78 \%$ of the time, whereas polygraph pen tracings were correct $60 \%$ of the time. However, it is not known to what degree observers would have been more accurate than speech EMG recordings under more typical conditions: (1) with less extremely high- and low-labial letters and with words; (2) during a delay period (or a stimulus presentation period) in which the observer cannot predict the frequency and timing of the stimulus occurrence; (3) in situations where there are more than two possibilities (i.e., high or low labial) and where the observers may have fewer than four chances (corresponding to each of the four phonemes) from which to make their choice. More information is needed about the value of this potentially money-saving technique.

The use of more sensitive control and data analysis procedures has enabled us to establish some important findings with regard to artifact-free subvocal speech. (1) Beyond any doubt, artifact-free subvocal speech does exist (Locke \& Fehr, 1970a, 1970b) and varies systematically with phoneme content of passages (Locke, 1971). (2) Contrary to previous assumptions derived from non-EMG research (Garrity, 1977), subvocal speech does in fact occur spontaneously in children as young as age 4 (Garrity, 1975a; Garrity \& Donoghue, 1977; Locke \& Fehr, 1970b). (3) Spontaneously occurring subvocal speech is in fact significantly related to recall, at least in preschool children (Garrity, 1975a; Garrity \& Donaghue, 1977). (4) Sex differences do exist, not in absolute amount of subvocal speech or of recall, but in the relationship between recall and subvocal speech: This relationship is significant for preschool boys but not for preschool girls (Garrity, 1975a; Garrity \& Donoghue, 1977). (5) Subvocal speech differences exist among preschool children: Younger, less bright preschoolers tend to subvocalize less when the stimuli are removed during the delay period than their older, less bright counterparts (Garrity, 1975a; Locke \& Fehr, 1970b). However, there tends to be no difference in amount of subvocalization between groups during stimulus presentation. This suggests that the increasingly higher correlations between recall and subvocal speech with increasing age reflect an increase primarily in rehearsal ability (or ability to maintain items in short-term memory), rather than in the ability to mediate phonologically (or to establish the phonological representation in short-term memory). (6) In addition, inhibition of subvocal speech results in reduced reading comprehension and in reduced recall, at least of difficult material. The above findings lend support to the moderately centralist theory of thought in which speech is, at a minimum, believed to index thought.

Given the present status of subvocal speech research, what appear to be the most promising future directions? (1) More descriptive studies relating subject variables to information processing differences are needed. For example, what is the relationship among sex, recall, and subvocal speech at different ages and IQ levels? Does EMG amplitude vary with age in a linear or curvilinear fashion? Tentative data indicate smaller amounts of speech EMG activity occur in younger 
preschoolers (Garrity \& Donoghue, 1977; Locke \& Fehr, 1970b), and in 10-year-olds (McGuigan \& Bailey, $1969 \mathrm{~b}$ ) compared to children aged 5 to 9 years. Do sex differences exist in EMG activity at different ages and IQ levels? How are subvocal speech frequency, patterning, and strategy affected by age, IQ, and sex?

(2) More studies are needed in which variables are experimentally manipulated. Few EMG studies in this area exist. The few that have attempted either to increase (McGuigan \& Pinkney, 1973) or decrease (Hardyck \& Petrinovich, 1969, 1970; Hardyck, Petrinovich, \& Ellsworth, 1966, 1967) the quantity of subvocal speech have met with limited success. Yet, it is only through such studies that it can be determined whether or not subvocal speech or rehearsal is causally related to recall, hence to short-term memory. In addition, few attempts have been made to manipulate more qualitative aspects of subvocal speech, such as to determine the effects of training in various amounts, patterns or strategies of rehearsal upon recall and upon amount of subvocal speech.

(3) More studies of children over wide age and IQ ranges are needed, particularly of a longitudinal nature. Child studies are important because of the light they can potentially shed upon the development of subvocal speech and its interrelationship with developing information processing abilities. Perhaps the only existing speech EMG longitudinal study of children reported a negative relationship between age and EMG amplitude (McGuigan \& Bailey, 1969b).

(4) More sophisticated and sensitive techniques are needed to record and analyze subvocal speech, particularly if more substantive information about patterns and strategies is to be found. Such techniques would, for example, enable differentiation of subvocalization of different words.

(5) More definitive information is needed on how subvocal speech is affected by different stimulus presentation procedures (aural, visual pictorial, visual linguistic) and by different recall (written, oral) and recognition (written, pictorial) procedures. Some evidence exists to indicate that significant amounts of subvocal speech are elicited by visually presented pictures and words (Goldstein et al.. 1972), by visually and aurally presented words (Locke \& Fehr, 1972), and by oral and written recall (Locke \& Fehr, 1971, 1972).

\section{REFERHNCES}

Bassin. F. V.. d Bein. E. S. Application of electromyography to the study of speech. In N. O'Connor (fid.). Recent Soriet psichology. New York: Liveright Publishing, 1961.

Camacho. E. O. Feedbalk of speech musele activity during silent reading: Tho comments. Sivence, 1967, 157. 581 .

Cole. R. A.. \& Young. M. Effect of subvocalization on menory for speech sounds. Journal of Experimental Psycholngy Human Learning and Memory. 1975. 1. $\because \div 2-7.4$.
EDFELDT. A. W. Silent speech and silent reading. Chicago: University of Chicago Press, 1959.

FAABORG-ANDERSEN. K. Electromyography of laryngeal nuscles in humans: Techniques and results. In $W$. F. Trojan (Ed.). Current problems in phoniatric and logopedics (Vol. 3). New York: S. Karger. 1965.

FaAborg-Andersen, K., \& Edfeld, A. W. Electronyography of intrinsic and extrinsic laryngeal muscles during silent speech: Correlation with reading activity. Acta Otolaryngologica. 1958, 49, 478-482.

Flavel.L. J. H., \& Hrl., J. P. Developmental psychology. Anmual Review of Psychology, 1969, 20. 1-56.

Garrity. L. I. An electromyographical study of subvocal speech and recall in preschool children. Developmental Psychology. 1975, 11, 274-281. (a)

GarRity, L. I. Measurement of subvocal speech: Correlations between two muscle leads and between two recording methods. Perceptual and Motor Skills, 1975, 40. 3273.30 . (b)

Garrity. L. I, A review of shorterm memory studies of cosert speech in young children. Jommal of Psuchology. $1977.95,249-261$.

Garrity, L. I.. \& Donoghue. J. T. A follow-up clectromyographical study of subvocal speech and recall in preschool children. Journal of Psychology. 1977. 95. $275-283$

Glassman. W. E. Subvocal activity and acoustic confusions in short-term memory. Joumal of Experimental Pswehology. 1972, 96, 164-169.

Goldstein, J., Locke, J. L.. \& Fehr, F. S. Children's pre-recall phonetic processing of pictures and printed words. Psychonomic Scionce, 1972, 26. 314-316.

Hardyck. C. D.. \& Petrinovich. L. F. Treatment of subvocal speech during reading. Journal of Reading. 1969. 12, $361-368.419-422$.

Hardyck, C. D., \& Petrinovich, L. F. Subvocal speech and comprehension level as a function of the difficulty level of reading material. Journal of Verbal Learning and Verbal Behavior. 1470. 9. 647-652.

Haroyck. (C. D.. Petrinovich, L. F.. \& Ellsworth, D. W. Feedback of speech muscle activity during silent reading: Rapid extinction. Science. 1966. 154. 1467-1468.

Hardyck. C. D.. Petrinovich, L. F.. \& Ellsworth, D. W. Feedback of speech muscle activity during silent reading: Two comments. Scionce, 1967, 157, 581.

Lippoid. O. C. J. Electromyography. In P. H. Venables \& 1. Martin (Eds.). A mamal of psychophysiological mothods. New York: American Elsevier. 1967.

Lorkf. J. L. Subvocial speech and speech. ASHA, 1970, 12. 7.14 .

Locke. J. L. Phonemic processing in silent reading. Perceprual and Motor Skills. 1971. 32. 905-906.

LoCKE, J. L.. \& FEHr. F. S. Subvocal rehearsal as a form of specoh. Jourmal of Verbal Learming and Verbal Behavior. 1970.9 .495 .498 . (a)

Locke, J. L.. \& Fehr, F. S. Foung children's use of the speech code in a recall task. Journal of Experimental Child Psichology'. 1970, 10, 367-37.3. (b)

Lockf, J. L., \& Fenr, F. S. Phonetic correlates of graphic recall. Percephul und Moner Skills. 1971, 33. 1040-1042.

Locke. J. L.. \& Fenr. F. S. Subvocalization of heard or seen words prior to spoken or written recall. American Journal of Psychology. 1972, 85, 6.3-68.

LOCKE. J. L.. \& GinsBerg, M. Electronyography and lip reading in the detection of verbal rehearsal. Bulletin of the Psychomomic Socioty. 1475, 5. 24h-248.

MCGuisan, F. J. Thinking: Studies of covert language behation. New York: Appleton-Century. 1466.

Mrciluin. F. J. Feedback of speed muscle activity during silent reading. Juo comments. Scienc', 1967. 157. 579-580. (a) 
MCGUIgan. F. J. Subrocal speech during silent reading. (Accession No. ED 015115) ERIC Document Reproduction Service. P. O. Drawer O. Bethesda. Md: 1967. (b)

MCGuigan. F. J. Covert oral behavior as a function of yuality of handwriting. American Journal of Psychology. 1970, 83, 377-388. (a)

McGuigan. F. J. Covert oral behavior during the silent performance of language tasks. Psychological Bulletin. 1970. 74. 309-326. (b)

McGuigan. F. J. Covert linguistic behavior in deaf subjects during thinking. Joumal of Comparative and Physiological Psychology, 1971, 75, 417-420.

McGuigan. F. J., \& Bailey. S. C. Covert response patterns during the processing of language stimuli. Interamerican Journal of Psychology. 1969. 3. 289-299. (a)

McGuigan, F. J.. \& Barley, S. C. Longitudinal study of covert oral behavior during silent reading. Perceptual and Motor Skills. 1969. 28. 170. (b)

McGuigan. F. J.. Culver. V. I.. \& Kendler. T. S. Covert behavior as a direct electromyographic measure of mediating responses. Conditional Reflex, 1971, 6. 145-152.

McGligan. F. J.. Keller. B.. \& Stanton. E. Covert language responses during silent reading. Journal of Educational Psichology. 1964. 55. 339-343.

McGuigan, F. J.. \& Pinkney, K. B. Effects of increased reading rate on covert processes. Interamerican Journal of Psichology. 1973. 7. 223-231.
McGuigan. F. J.. d Rodier, W. I.. III. Effects of auditory stimulation on covert oral behavior during silent reading. Journal of Experimental Psychology. 1968, 76, 649-655.

McGuigan. F. J.. \& Winstead, C. L. Discriminative relationship between covert oral behavior and the phonemic system in internal information processing. Journal of Experimental Psychology. 1974, 103, 885-890.

Novikova, L. A. Electrophysiological investigation of speech. In N. O'Connor (Ed.), Recent Soviet psychology. New York: Liveright. 1961.

Sokolov, A. N. Speech-motor afferentiation and the problem of brain mechanisms. Soviet Psychology, 1967. 6. 3-15.

Sokolov. A. N. Studies of the speech mechanisms of thinking. In M. Cole \& I. Maltzman (Eds.), A handbook of contemporun Soviet psychology. New York: Basic Books. 1969.

Sokolov. A. N. Inner speech and thought. New York: Plenum Press. 1972.

Vygotsky, L. S. Thought and language. Cambridge, Mass: MIT Press. 1962.

(Received for publication September 7, 1977; accepted September 12, 1977.) 